\title{
Application of the Bispectral Wavelet Analysis for Searching Three-Wave Interactions in the Spectrum of Internal Waves
}

\author{
G. V. Zhegulin ${ }^{1, \otimes}$, A. V. Zimin ${ }^{1,2}$ \\ ${ }^{1}$ Shirshov Institute of Oceanology, Russian Academy of Sciences, Moscow, Russian Federation \\ ${ }^{2}$ Saint-Petersburg State University, Saint-Petersburg, Russian Federation \\ 凶leb-jegulin@rambler.ru
}

Purpose. The aim of the work is to test the bispectral wavelet analysis being applied as a tool for studying resonance interactions between the frequency components in the spectrum of internal waves (based on the example both of the model signals, the shape of which is similar to that of the solitons and boras, and the field observations data on temperature fluctuations resulted from the internal waves in the Gorlo Strait of the White Sea).

Methods and Results. The paper represents a technique for detecting three-wave interactions in the internal waves' spectrum. The method is based on the bispectral wavelet analysis. It permits to identify the interharmonic correlation and the magnitude of the quadratic phase relationship arising as a result of nonlinear interactions between the signal frequency components. In the first part of the paper, efficiency of the applied method was evaluated using the example of various artificial signals with quadratic nonlinearity in order to demonstrate the method features and advantages. In its second part, the method was used to analyze the temperature profiles obtained by scanning thermohaline sounding, in which the oscillations related to passing of the internal wave groups were recorded. It is shown that the waves with the $40 \mathrm{~min}$ period are generated due to quadratic nonlinearity. The auto-bicoherence function values confirm the fact that the higher harmonics are formed in the 60-120 min range as a result of the three-wave interactions. They change synchronously in time, and their amplitudes are proportional, that is typical of the initial stage of the waves' nonlinear transformation. Absence of a periodic change in the biphase sign in the considered range indicates insignificant influence of the dispersion effects upon the short-period internal waves' structure.

Conclusions. The example of observations in the Gorlo Strait of the White Sea shows that the recorded asymmetric structure of the isotherm oscillations was formed being influenced by the three-wave interaction. Possibility of further application of the method for studying the processes of the internal waves' nonlinear transformation and breaking is discussed.

Keywords: temperature fluctuations, internal waves, nonlinearity, auto-bicoherence, phase coupling, asymmetry, three-wave interaction

Acknowledgments: the work was carried out within the framework of the state task on theme No. 0128-2021-0014 "Wave processes, transport phenomena and biogeochemical cycles in the seas and oceans: a study of the forming mechanisms based on physical and mathematical modeling and field experimental work". The authors are especially grateful to Justin A. Schulte (http://justinschulte.com/) for the algorithm developed on the basis of modern statistical methods for analyzing unsteady signals, and for the software packages accessed free in the network, that significantly helped in preparing the paper.

For citation: Zhegulin, G.V. and Zimin, A.V., 2021. Application of the Bispectral Wavelet Analysis for Searching Three-Wave Interactions in the Spectrum of Internal Waves. Physical Oceanography, [e-journal] 28(2), pp. 135-148. doi:10.22449/1573-160X-2021-2-135-148

DOI: $10.22449 / 1573-160 X-2021-2-135-148$

(c) G. V. Zhegulin, A. V. Zimin, 2021

(C) Physical Oceanography, 2021 


\section{Introduction}

For the analysis of stationary signals, the methods of classical spectral analysis, such as fast Fourier transform or cross-spectral analysis, are sufficient as a rule, while it is assumed that the variable is distributed according to the Gaussian law [1, 2]. Spectral power density estimates are useful in determining the contribution of each spectral component to the overall spectrum of a time series signal. However, the traditional analysis based on the second-order spectra obtained using the classical Fourier transform is inapplicable for non-stationary signals with time scales that are much smaller than the realization length of the signal under study. At the same time, local changes make almost no contribution to the resulting spectrum. For example, the signals corresponding to nonlinear internal waves (IWs), such as solitons and boras, have an asymmetric and sawtooth shape. Accordingly, such oscillations are described by statistics of a random process, different from Gaussian ${ }^{1}$ ones. The most obvious method for studying the spectral properties of such processes is the wavelet analysis. For example, when using complex functions, continuous wavelet transform provides the study of dynamics of such characteristics as instantaneous frequencies, instantaneous amplitudes and instantaneous phases of rhythmic processes identified in the structure of the analyzed signal.

In order to analyze the nonlinear IWs, one can use more complex statistical characteristics of signals, such as higher-order spectra (poly-spectra) [3], obtained using Fourier transforms of cumulant functions ${ }^{2}$. In this case, spectral characteristics of odd orders, for example, a spectrum of the third order (bispectrum ), which is the Fourier transform of the third-order cumulant function, can be useful for refining the asymmetry parameters of the signal under study [4]. When studying symmetric nonlinearities, a more powerful tool is the fourth-order spectrum (trispectrum), since it is a frequency expansion of kurtosis [5]. Spectral analysis based on higher-order spectra makes it possible to quantify nonlinearities, characterize their types, and detect interacting harmonics within a time series, which is especially important for studying the characteristics of real internal waves.

The expediency of using polyspectra is justified by the ability to extract important information about the coherent relationships of pairs of spectral components in the observed process, which is lost when assessing the energy spectrum.

Bispectral wavelet analysis provided not only the identification of interharmonic correlation, but also the investigation of temporal dynamics of the phase relationship between certain components in complex nonstationary signals. The normalized bispectrum (bicoherence) characterizes the phase relationships (phase connection) between different frequency components of the signal [6]. The bispectrum of a process with an asymmetric distribution law is not zero. This allows bispectral analysis to be used as a sensitive indicator of nonlinear processes.

When the signal contains two harmonics with $f_{1}$ and $f_{2}$ frequencies simultaneously with their sum $f_{3}$, and the sum of the phases of these harmonics remains constant, we can talk about the phase relationship. Such a set of frequency components is called bispectrally organized triplet. Correspondingly, the bicoherence

\footnotetext{
${ }^{1}$ Malakhov, A.N., 1978. [Cumulant Analysis of Random Non-Gaussian Processes and Their Transformations]. Moscow, Sovetskoe radio Publ., 376 p. (in Russian).

${ }^{2}$ Botchkov, G.N. and Gorokhov, K.N., 2007. [Polyspectral Analysis and Synthesis of Signals]. Nizhny Novgorod: Lobachevsky State University of Nizhny Novgorod, 113 p. (in Russian).
} 
function will be close to unity when the signal contains frequencies satisfying the relation

$$
f_{1}+f_{2}=f_{3} .
$$

If the relation (1) is not satisfied, the bicoherence function tends to zero [6, 7].

The use of bispectral and trispectral analysis (especially based on wavelet analysis) in hydrometeorology is significantly limited, while second-order spectral analysis using a time-frequency or dual-frequency spectrum is often used for hydrometeorological data [8]. The use of bicoherent wavelet analysis to detect phase relationships in the structure of wind-wave interaction is considered in [9]. However, the study did not test for statistical significance. D. Schulte [10] consistently and thoroughly considered the identification of nonlinear interactions in an artificially generated signal and quasi-biennial oscillation of the zonal wind in the equatorial stratosphere. In this work, the spectra of local autobicoherence and biphase were also calculated with the estimates of confidence intervals and significance levels. Nonlinear changes in climatic processes have occurred many times and have had a serious impact on ecological and economic systems [11]. However, such an analysis has not previously been used to study short-period internal waves (SIW).

The purpose of this work is to test the application of auto-bicoherent wavelet analysis as a tool for studying nonlinear resonant interactions between frequency components in the spectrum of internal waves and identifying the phase relationship between harmonics.

At the first stage, the efficiency of the method used in this work was estimated on the data of a model signal in a medium with quadratic nonlinearity in order to study in detail the properties of the auto-bicoherent wavelet transform and to clarify the method of its application to the analysis of nonstationary signals (see also [10, 12]), and on the second stage it was based on the results of experimental observations of the oscillations of isotherms near the pycnocline obtained in the White Sea Throat in August 2012.

\section{Research method}

Details of the method used in this work and its fundamental foundations can be found in $[6,7,10,12]$. Here we give only key concepts and important illustrations.

By analogy with the Fourier bispectrum, the wavelet mutual autobispectrum has the following form $[7,9,10]$

$$
B_{x x x}^{W}\left(s_{1}, s_{2}\right)=\int_{T} W_{x}^{*}(s, t) W_{x}\left(s_{1}, t\right) W_{x}\left(s_{2}, t\right) d t,
$$

where

$$
\frac{1}{s_{1}}+\frac{1}{s_{2}}=\frac{1}{s}
$$

$T$ is time interval; $s$ is a time scale of the independent harmonic component resulting from the phase relationship between the harmonics with $s_{1}$ and $s_{2}$ scales; $W_{x}(s, t)$ is a wavelet transform of $x$ time series, and the asterisk in $W_{x}^{*}(s, t)$ denotes a complex conjugation operation. Accordingly, autobicoherence is defined as a normalized autobispectrum $[6,10]$ : 


$$
b_{x x x}^{W}\left(s_{1}, s_{2}\right)=\frac{\left|B_{x x x}^{W}\left(s_{1}, s_{2}\right)\right|^{2}}{\left(\int_{T}\left|W_{x}\left(s_{1}, t\right) W_{x}\left(s_{2}, t\right)\right|^{2} d t\right)\left(\int_{T}\left|W_{x}(s, t)\right|^{2} d t\right)^{\prime}},
$$

where $b_{x x x}^{W}\left(s_{1}, s_{2}\right)$ varies within the range from 0 to 1 .

In practice, quadratic nonlinearity leads to the fact that, as a result of the interaction of two harmonics of the process, part of the power is released at the total and (or) difference frequencies of these components. Wavelet-based autobicoherence determines the value of the nonlinear coupling between harmonics, where a peak in $\left(s_{1}, s_{2}\right)$ plane indicates a statistical relationship between components with scales $s_{1}, s_{2}$ and $s$.

Autobispectrum allows you to introduce a function called biphase, which is defined as

$$
\psi=\operatorname{tg}^{-1}\left(\frac{\operatorname{Im}\left(B_{x x x}^{W}\left(s_{1}, s_{2}\right)\right)}{\operatorname{Re}\left(B_{x x x}^{W}\left(s_{1}, s_{2}\right)\right)}\right)=\varphi_{1}+\varphi_{2}-\varphi_{3} .
$$

Biphase characterizes the variation in signal asymmetry. A value of $\psi$ equal to zero indicates a positive asymmetry, equal to $180^{\circ}$ - a negative one [12]. In fact, if $|\psi|>90^{\circ}$, then the waveform shifts to the region of negative values. In this case, the signal is said to have negative distortion.

In order to demonstrate the properties of the biphase in the same way as it was done in $[10,12]$, we consider a time signal similar to the manifestations of various nonlinear SIWs in the form

$$
\left\{\begin{array}{c}
X(t)=\sum_{j=1}^{40} \frac{1}{j} \cos [0,1 j t+\gamma(j-1)], \quad \text { где } \gamma=0 ; \pm \frac{\pi}{2} ; \pm \pi ; \\
\xi_{X}(t)=\frac{X(t)-\mu_{X}}{\sigma_{X}}
\end{array}\right.
$$

where $\mu_{X}$ is an arithmetic mean; $\sigma_{X}$ is a standard deviation, and $\xi_{X}(t)$ is a normalized value of each element of averaged time series $X(t)$. The transformation of the averaged time series with its reduction to zero mean and unit variance (normalization) is performed in order to obtain a single numerical scale.

In Fig. 1, $a$ an oscillation visually similar to the manifestation of a group of nonlinear internal waves of elevations is given. It can be seen that the deviations from the mean in the positive direction of the vertical axis are sharper, but less frequent than in the negative direction. In this case, the biphase oscillates around $0^{\circ}$. In Fig. $1, d$ a signal similar to a group of internal waves of depressions, similar to those presented in [13]. In this case, the type of biphase is fundamentally different from that in Fig. 1, $a$ : it is close to $\pm 180^{\circ}$. 

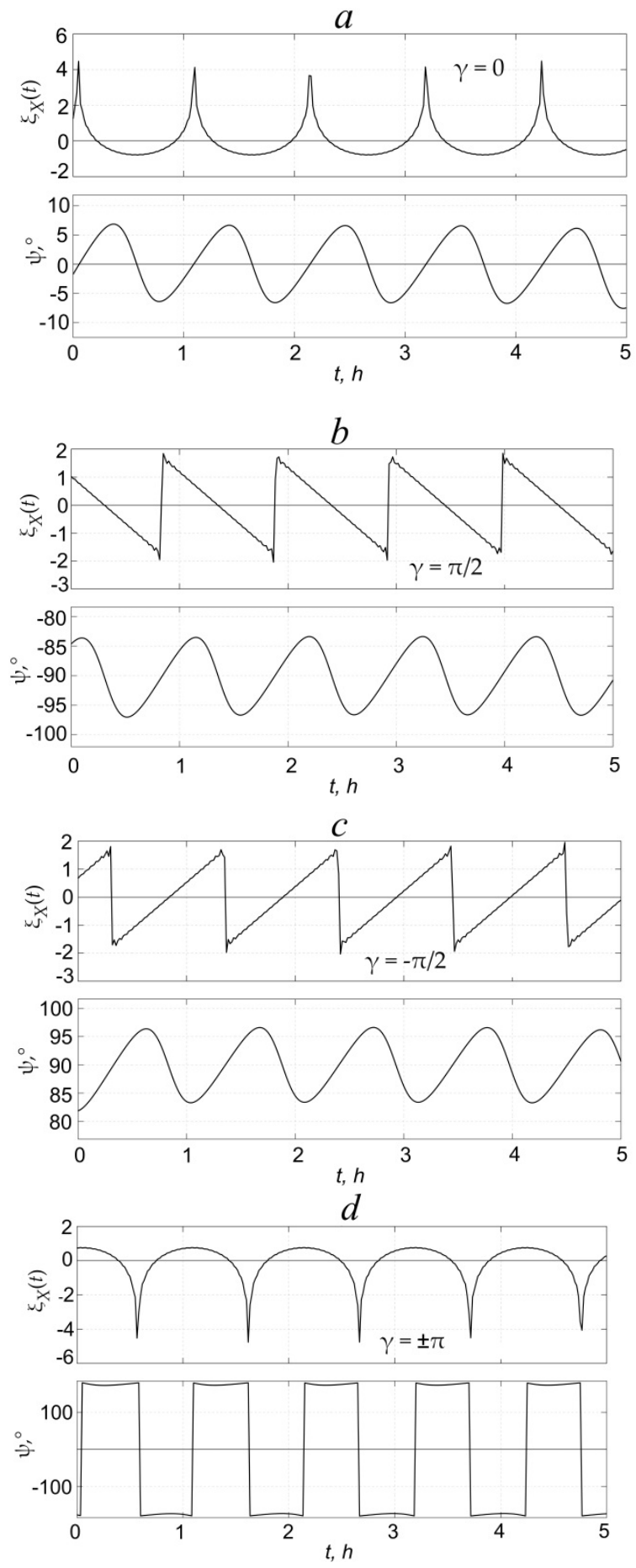

F i g. 1. Normalized time series $X(t)$ and its local biphase $\psi$ for various values of $\gamma$ in expression (3): $\gamma=0(a) ; \gamma=\pi / 2(b) ; \gamma=-\pi / 2(c) ; \gamma= \pm \pi(d)$. The biphase was calculated for a triplet with the time scales $s_{1}=60, s_{2}=30$ and $s_{3}=20 \mathrm{~min}$ 
The biphase for oscillations with different anterior and rear fronts is represented in Fig. 1, $b$ and $c$. A completely asymmetric time series at $\gamma= \pm \pi / 2$ in expression (3) will have a biphase of $90^{\circ}$ or $-90^{\circ}$.

\section{Autobigoherent wavelet analysis of model signal}

In order to study the properties of the auto-bicoherent wavelet transform and refine the methodology for its application to the analysis of multifrequency signals, for example, similarly to how it was done in [10,12], we will consider another model time signal (similar to an internal eagry) with the addition of white Gaussian noise $\omega(t)$ :

$$
\left\{\begin{array}{r}
Y(t)=\sum_{j=1}^{100} \frac{1}{j} \cos \left[0,1 j t-\frac{3 \pi}{2}(j-1)\right]+\omega(t), \\
\xi_{Y}(t)=\frac{Y(t)-\mu_{Y}}{\sigma_{Y}},
\end{array}\right.
$$

where $\mu_{Y}$ is mean; $\sigma_{Y}$ is a standard deviation and $\xi_{Y}(t)$ is a normalized value of each element of averaged time series $Y(t)$.

For the analysis, the Morlet wavelet was chosen as the parent wavelet, which makes it possible to achieve better frequency localization and estimate the value of the frequencies of the multicomponent signal with a lower error compared to other basic wavelets $[6,14]$.

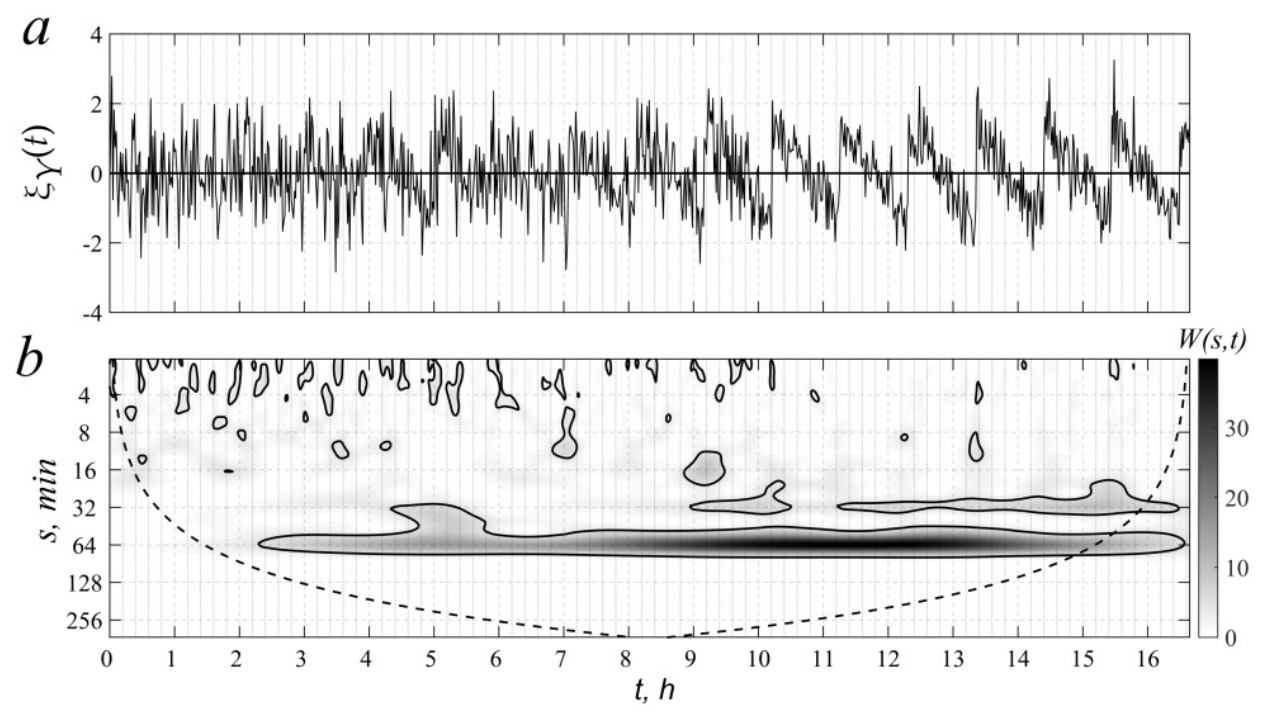

F i g. 2. Results of a continuous wavelet transform of the normalized time series (4) with addition of white noise of different intensities $(a)$, and the local wavelet spectrum $(b)$. The dashed line separates the region of the edge effects, the bold lines show the 5\%-significance level in relation to red noise 
The normalized time sequence $Y(t)$ and its local wavelet spectrum $W(s, t)[13$, 15 ] for the case of degenerate interaction, when the condition $f_{1}=2 f_{2}$ is satisfied, are presented in Fig. 2. This process is just formally a three-wave one, since in fact two waves are involved in it. The frequencies of them differ by two times. The main wave $f_{1}$ lies in a large region of $5 \%$ significance at $s_{1}=60 \mathrm{~min}$ in the recording section between 3 and $16 \mathrm{~h}$, and its second harmonic $f_{2}$ lies in two small regions of significance in the same time interval. Note that the occurrence of statistically significant values of the spectrum at $s=30 \mathrm{~min}$ in the range $t=9 \div 16 \mathrm{~h}$ is accompanied by asymmetry (see Fig. 2, a). For assessing the level of significance, the Monte Carlo method was applied, as in $[10,16]$.

To determine whether the oscillations are quadratically nonlinear, an autobicoherence was calculated (Fig. 3, a). From Fig. 3, $a$ it can be seen that the region of statistical significance contains a local maximum of auto-bicoherence (marked with a red asterisk) on time scales $s_{1}=50 \mathrm{~min}$ and $s_{2}=75 \mathrm{~min}$ and indicates that oscillations with periods of 50 and $75 \mathrm{~min}$ are phase-related with the oscillations of $s=30$ min period The variability of the time scales $s$ is partly due to the statistical relationship between the three harmonics. In this case, $b_{x x x}^{W}\left(s_{1}, s_{2}\right) \approx 0.85$, so that approximately $85 \%$ of the variability at $s=30 \mathrm{~min}$ is due to the nonlinear interaction between the harmonics, the time scales of which can be determined from the location of the maximum on the plane $\left(s_{1}, s_{2}\right)$.
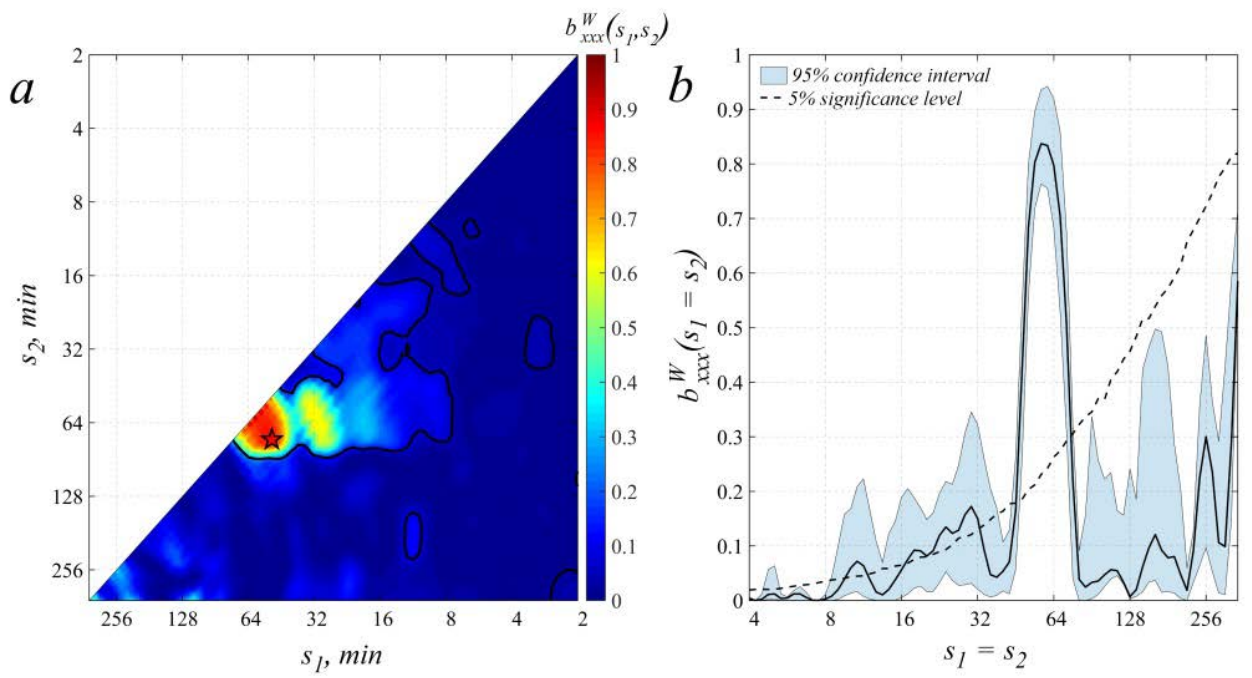

F i g. 3. Auto-bicoherence of the model time series (4) (a); the diagonal cross section of the autobiherence spectrum for $s_{1}=s_{2}(b)$. The bold lines contour the 5\%-significance areas in relation to red noise. The diagonal line divides the spectrum into two symmetric regions. The significance level represented by the dashed line was calculated using the Monte Carlo method. The confidence intervals were determined by the boot method (bootstrapping) [10, 17]

In Fig. 3, $b$ the result of applying the bootstrapping procedure [10, 17] to the diagonal section $s_{1}=s_{2}=s$ of the autobicoherent spectrum $b_{x x x}^{W}\left(s_{1}=s_{2}\right)$ for 
the case of degenerate three-wave interaction. The basic principle of bootstrapping is to simulate multiple Monte Carlo sampling from an general population using the data from an existing sample. A brief overview and detailed description of the method can be found in [10, 17-19]. In order to determine the confidence intervals for auto-bicoherence, at each time scale, the set of wavelet coefficients was divided into overlapping segments (blocks) of equal length. The subsequent merging of the blocks leads to a synthetic set of wavelet coefficients at each time scale. Iteration of the procedure for 3000 times results in a distribution of bootstrap retries, from which a 95\% confidence interval can be obtained. The appropriate block length was determined by the Monte Carlo method $[10,16,18]$ in order to find the largest $c$ value at which the block length $L=N^{c}$, where $N$ is the length of the original series, would give the widest confidence intervals. It was revealed that the block length within the range from $N^{0.6}$ to $N^{0.7}$ is optimal for estimating the confidence intervals [10].

Fig. 3, $b$ it can be seen that the width of the confidence intervals does not depend on the time scale. The confidence interval of $95 \%$, corresponding to the maximum of auto-bicoherence with the time scales $s_{1}=s_{2}=60 \mathrm{~min}$, does not cross $5 \%$ significance limit, and, therefore, this peak is significant. All other peaks were considered insignificant.

Periodic energy exchange between harmonics can significantly affect the biphase evolution. Therefore, in order to track the temporal variability of the intensity of three-wave interactions, local auto-bicoherence is calculated [10]:

$$
b_{t}^{W}\left(s_{1}, s_{2}\right)=\frac{\left|S\left(s_{1}^{-1} B_{t}^{W}\left(s_{1}, s_{2}\right)\right)\right|^{2}}{S\left(s_{1}^{-1}\left|W_{t}^{x}\left(s_{1}\right) W_{t}^{x}\left(s_{2}\right)\right|^{2}\right) \cdot S\left(s^{-1}\left|W_{t}^{x}(s)\right|^{2}\right)}
$$

where $S$ is a smoothing operator for Morlet wavelet [10, 15].

The local auto-bicoherence spectrum of signal (4) with Gaussian noise for a bispectrally organized triplet $\left(s_{1}=50 \mathrm{~min}, s_{2}=75 \mathrm{~min}, s=30 \mathrm{~min}\right.$ ) is given in Fig. 4, $b$.

From Fig. 4 it can be seen that after 7 h the quadratic nonlinearity is strong: it leads to the formation of a region of significant local auto-bicoherence extended in time. A mutual growth of the $1^{\text {st }}$ and $2^{\text {nd }}$ harmonics is noted, the resonant interaction of which with the $3^{\text {rd }}$ leads to an asymmetric signal structure. The biphase in this section becomes sharply negative and stable, which indicates constant phase dependence between the harmonics. The region of a statistically significant autobicoherence section, exceeding 0.8 (see Fig. 3, a), covers many resonant triplets from 40-70 min range. In this case, the biphase is invariant for all sets of harmonic pairs. The biphase proximity to $-90^{\circ}$ indicates a complete asymmetry of the signal - a sawtooth shape with a sharp leading edge (see Fig. 1, b). High and statistically significant estimates of auto-bicoherence (over 0.8 ) indicate that the $1^{\text {st }}$ and $2^{\text {nd }}$ harmonics are related for a certain period of time. 

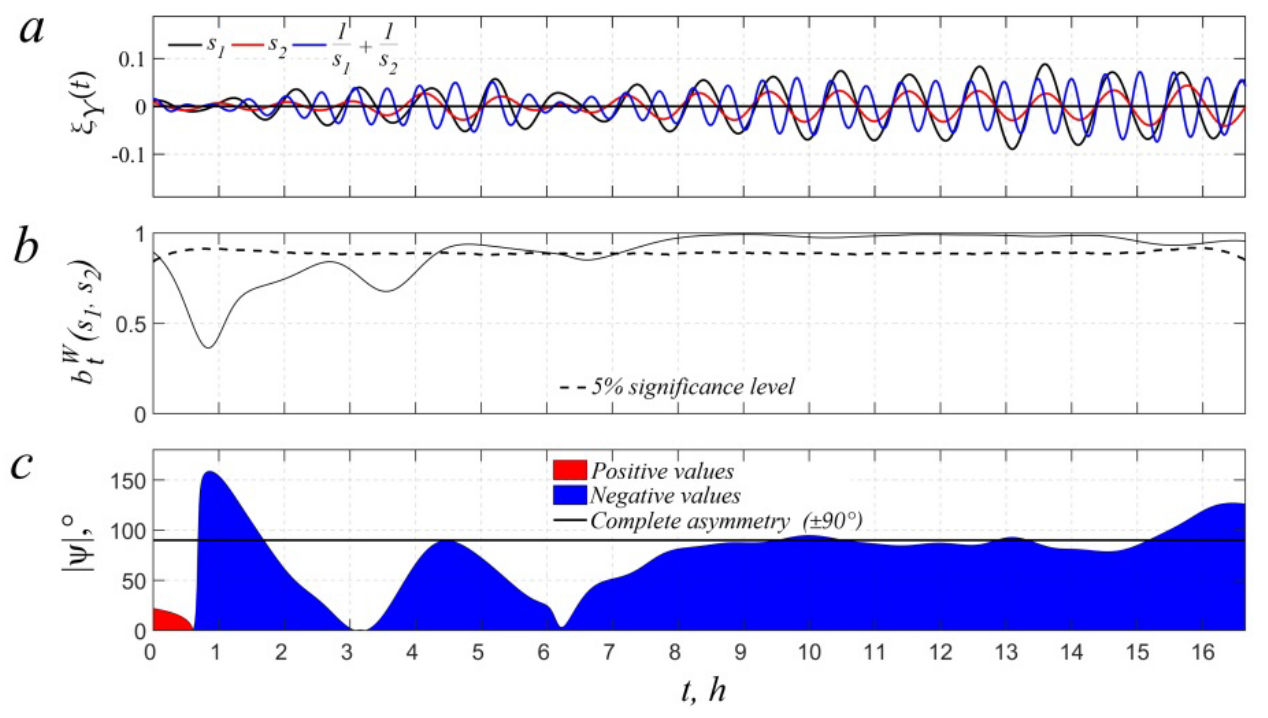

F i g. 4. Harmonics of the resonance bispectral triplet (50, 75, $30 \mathrm{~min})$ ( $a$ ), local auto-bicoherence (b) and local biphase (c) of the auto-bicoherence spectrum (Fig. 3, $a$ ) at the time scales 50 and $75 \mathrm{~min}$. The biphase oscillations differing from $0^{\circ}$ indicate that the nonlinear interaction led to a waveform with asymmetry

\section{Analysis of temperature variations in the White Sea Throat}

The data of scanning measurements in oceanology can be considered as a twodimensional picture of variations in hydrophysical fields along the vertical over time, which gives a visual representation of the variability of processes. In Fig. 5 sharp short-period temperature fluctuations in the thermocline region, which in [20] were identified as packets of intense SIWs with nonlinearity signs, are presented. In the example, the IWs observations were considered. They were carried out from a moored vessel by a CTD-probe in the mode of continuous scanning from the surface to the bottom with 1-2 min discreteness at a depth of about $50 \mathrm{~m}$. The observation duration of each of the intensive IW packets varied from 40 to $120 \mathrm{~min}$. The packets moved in one direction to the southwest, which was revealed from the data of synchronous with contact satellite observations, the results of which are presented in [21]. At the same time, the occurrence depth and the volumes of warm and cold waters in the section plane experienced significant fluctuations in other periods as well. Based on these field data, the method of obtaining and the results, the processing of which are described and analyzed in detail in [20], the method was tested.

A preliminary analysis of temperature variations was carried out using a Savitzky - Golay filter with 180 min window in order to exclude the influence of long-period oscillations. Then the numerical series were normalized. 


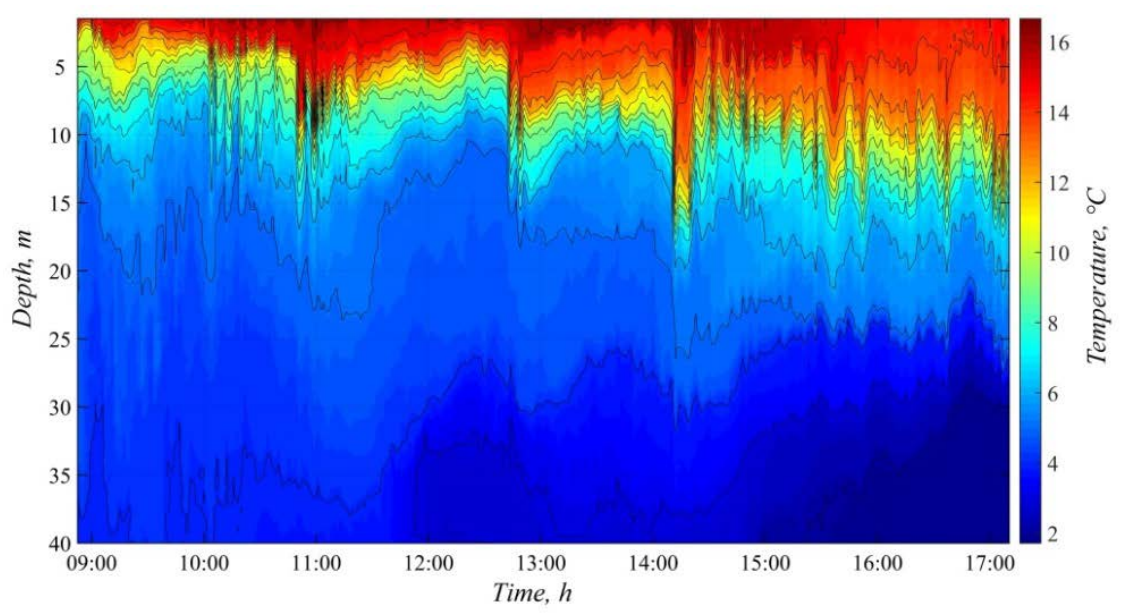

F i g. 5. Time series of temperature at depths of $1.5-40 \mathrm{~m}$ in the White Sea Throat, taken with a time interval of $1 \mathrm{~min}$, and at a depth of $0.5 \mathrm{~m}$ in the period from 08:54 to 17:10 (01.08.2012)

In order to search for nonlinear resonance interactions in the IWs spectrum, auto-bicoherence was calculated for each horizon. An area with increased values of auto-bicoherence was identified (more than 0.8 ) in the transition layer at 5-20 m depths. For example, the auto-bicoherence value at $10 \mathrm{~m}$ depth with $s_{1}=s_{2}=80$ min time scales (see Fig. 6, $a$ ) indicates on a statistically significant nonlinear relationship of the degenerate three-wave interaction type. The region of confidence intervals (see Fig. 6, b) does not intersect with 5\% boundary of statistical significance near 80 min scale. Therefore, we can confidently speak about the generation of the second harmonic with 40 min period.

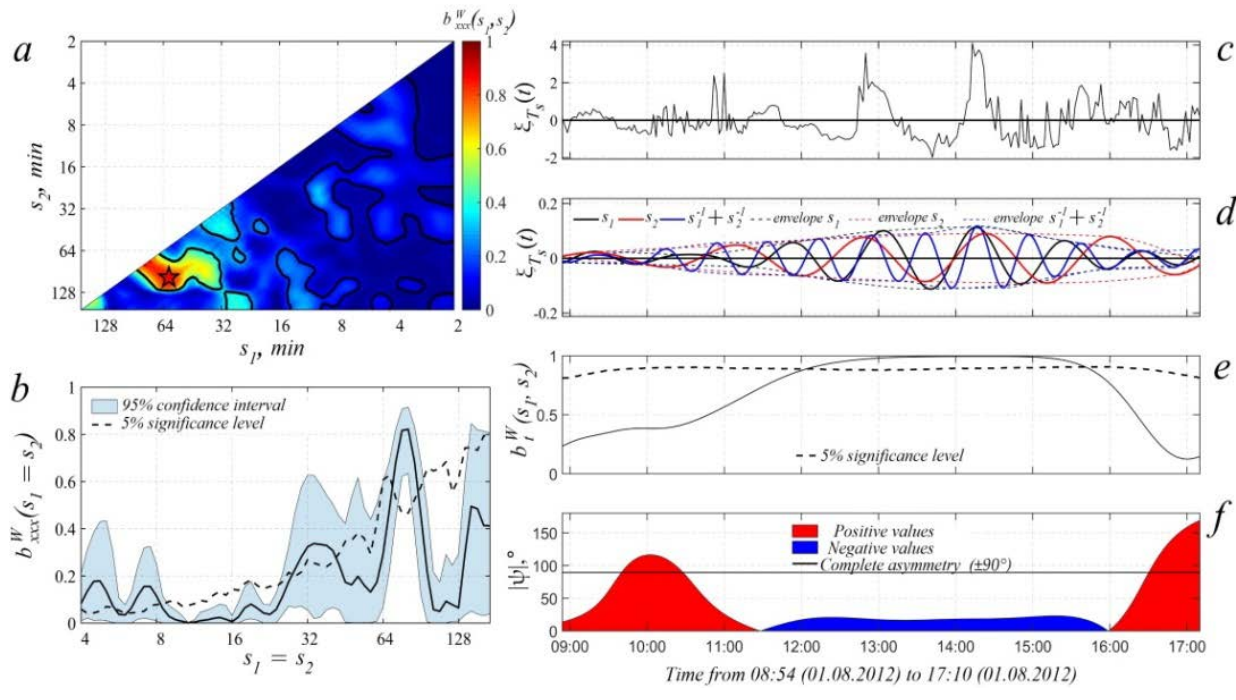

F i g. 6. Auto-bicoherent wavelet analysis of temperature fluctuations at the depth $10 \mathrm{~m}: a$ - autobicoherence; $b$ - diagonal section of the auto-bicoherence spectrum; $c$ - normalized temperature pulsations; $d$-harmonics of the resonance triplet (60, 100, $40 \mathrm{~min}) ; e$ - local auto-bicoherent spectrum; $f$ - local biphase spectrum 
The local auto-bicoherence spectrum of temperature fluctuations with $s_{1}=$ $60 \mathrm{~min}$ and $s_{2}=100 \mathrm{~min}$ (see Fig. 6,e) time scales indicates when the interaction takes place, for example, in the period from 12:00 to 15:45. Asymmetric distortions of the signal structure (see Fig. 6, $c$ ) in this time interval are the result of the steadystate resonant interaction between the harmonics of 60, 100, and 40 min. However, it is worth noting that the area of the significant auto-bicoherence section, exceeding 0.8 (see Figure 6, $a$ ), covers many sets of harmonic pairs from 60-120 min range, participating in three-wave interactions.

In Fig. 6, $f$ the biphase evolution corresponding to a local peak of autobicoherence with $s_{1}=60 \mathrm{~min}$ and $s_{2}=100 \mathrm{~min}$ time scales is presented. From 9:00 to $12: 00$, the graph $6, f$ represents unstable phase dependence between the components of the resonance triplet. This lack of phase coherence is consistent with a low level of auto-bicoherence (see Fig. 6, $e$ ) in this region. That is, there is no three-wave interaction here. On the contrary, from 12:00 to 15:45 the biphase is stable and ranges from -20 to $-25^{\circ}$. It can be concluded that the asymmetric structure of oscillations here was due to the result of nonlinear interaction between the harmonics.

The quantitative biphase assessment for the transition layer $(8-18 \mathrm{~m})$ in the period from 12:00 to 15:45, as well as the local auto-bicoherence for other triplets from 60-120 min range, is stable. In this layer, the maximum values of autobicoherence are noted, and about $80 \%$ of the biphase values for the triplet $(60,100$, $40 \mathrm{~min}$ ) lie within the range from 0 to $-25^{\circ}$. During the three-wave interaction, the energy is exchanged between the waves, due to which the amplitudes periodically change, as shown, for example, in [22]. In the given example, the biphase is preserved, the envelopes of the harmonics vary synchronously with time and their amplitudes are proportional (Fig. 6, $d, e$ ), respectively, according to the conclusions of [23], the periodic energy exchange is not observed in our case. The absence of a periodic change in the biphase sign within the range under consideration indicates a weak influence of dispersion effects on the SIWs structure. This is also confirmed by the estimates of the dispersion relation for internal waves, made from the data of hydrological observations in [24].

In addition, in the considered example of field data with the biphase negative values, the mode of complete asymmetry of the signal, as, for example, in Fig. 1, $b$ is not observed. Therefore, the IWs, despite their nonlinear character, most likely were not in the stage of local instability or breaking and could propagate far enough. This is indirectly confirmed by the results of [25], in which it was demonstrated that IWs packets, arising regularly (each tidal cycle) in the southern part of the White Sea Throat, move across the entire sea in a southwestern direction, transforming during propagation, and finally disintegrate only after $200 \mathrm{~km}$ in the shallow water zone northwards of the Solovetsky archipelago.

Manifestations of nonlinear transformation and breaking processes were considered in [23, 26], in which the influence of wave breaking on the biphase spatial variability was studied in relation to surface waves in the coastal zone. According to $[27,28]$, the surface waves near the coast before the breaking take a sawtooth shape and the biphase in them is close to $-\pi / 2$. An example of IWs at the breaking stage is a model signal with Gaussian noise (see Fig. 2, $a$ ), where, from 9 h, the biphase becomes close to $-\pi / 2$, and the signal takes a sawtooth shape (see Fig. $4, c$ ). PHYSICAL OCEANOGRAPHY VOL. 28 ISS. 2 (2021) 
However, an assessment of the processes of IWs local instability and breaking based on auto-bicoherent wavelet analysis has not been described in the literature. Therefore, it seems appropriate to continue further consideration of this issue, which is important for automated processing of data on thermocline oscillations.

\section{Conclusion}

This paper describes the application of a technique based on bispectral wavelet analysis, which provides the detection of nonlinear interactions, to the study of the characteristics of the internal waves.

On the model and field examples, the possibility of higher-order statistics application, based on wavelet analysis, to study the processes of nonlinear transformation of internal waves is presented.

Auto-bicoherent wavelet analysis was applied for the first time to detect the nonlinear interactions of internal waves observed in temperature time series in the White Sea Throat.

Analysis of temperature fluctuations provided to reveal the relationship between harmonics in a certain period of time. It is shown that the relationship between frequency components was accompanied by the distortion of asymmetric signal.

High statistically significant values of auto-bicoherence (more than 0.9 ) indicate that harmonics within the range of $60-120 \mathrm{~min}$ are the waves coupled due to threewave interactions. The biphase value during the period of three-wave interaction intensification was predominantly within the range from -20 to $-25^{\circ}$. The absence of a periodic change in the sign of the biphase confirms the insignificant influence of the dispersion effects observed in the southern part of the White Sea Throat on the structure of the SIWs signal.

Summarizing the work results, it should be noted that the widespread use of polyspectral analysis methods will significantly expand the understanding of hydrophysical processes associated with the internal waves in the ocean.

\section{REFERENCES}

1. Jenkins, G.M. and Watts, D.G., 1971. Spectral Analysis and Its Applications. San Francisco: Holden Day, 1968, 525 p.

2. King, T., 1996. Quantifying Nonlinearity and Geometry in Time Series of Climate. Quaternary Science Reviews, 15(4), pp. 247-266. https://doi.org/10.1016/0277-3791(95)00060-7

3. Novikov, A.K., 2002. [Multispectral Analysis]. St. Petersburg, 180 p. (in Russian).

4. Zelensky, A.A., Kravchenko, V.F., Pavlikov, V.V. and Totsky, A.V., 2013. Bispectrum Analysis in Digital Signal Processing and Applications. Physical Bases of Instrumentation, 2(3), pp. 4-39 (in Russian).

5. $\quad$ Collis, W.B., White, P.R. and Hammond, J.K., 1998. Higher-Order Spectra: the Bispectrum and Trispectrum. Mechanical Systems and Signal Processing, 12(3), pp. 375-394. https://doi.org/10.1006/mssp.1997.0145

6. Koronovsky, A.A. and Khramov, A.E., 2003. [Continuous Wavelet Analysis and Its Applications]. Moscow: Fizmatlit, 176 p. (in Russian).

7. Van Milligen, B.Ph., Sánchez, E., Estrada, T., Hidalgo, C., Brañas, B., Carreras, B. and Garcìa, L., 1995. Wavelet Bicoherence: A New Turbulence Analysis Tool. Physics of Plasmas, 2(8), pp. 3017-3032. https://doi.org/10.1063/1.871199

8. Rozhkov, V.A., 1979. [Probabilistic Analysis Methods for Oceanic Processes]. Leningrad: Gidrometeoizdat, 280 p. (in Russian). 
9. $\quad$ Elsayed, M.A.K., 2006. Wavelet Bicoherence Analysis Of Wind-Wave Interaction. Ocean Engineering, 33(3-4), pp. 458-470. https://doi.org/10.1016/j.oceaneng.2005.04.013

10. Schulte, J.A., 2016. Wavelet Analysis for Non-Stationary, Nonlinear Time Series. Nonlinear Processes in Geophysics, 23(4), pp. 257-267. https://doi.org/10.5194/npg-23-257-2016

11. Alley, R.B., Marotzke, J., Nordhaus, W.D, Overpeck, J.T., Peteet, D.M., Pielke Jr., R.A., Pierrehumbert, R.T., Rhines, P.B., Stocker, T.F., Talley, L.D. and Wallace, J.M., 2003. Abrupt Climate Change. Science, 299(5615), pp. 2005-2010. https://doi.org/10.1126/science.1081056

12. Maccarone, T.J., 2013. The biphase Explained: Understanding the Asymmetries in Coupled Fourier Components of Astronomical Time Series. Monthly Notices of the Royal Astronomical Society, 435(4), pp. 3547-3558. https://doi.org/10.1093/mnras/stt1546

13. Serebryany, A.N. and Pao, H.P., 2008. Transition of a Nonlinear Internal Wave through an Overturning Point on a Shelf. Doklady Earth Sciences, 420(1), pp. 714-718. https://doi.org/10.1134/S1028334X08040430

14. Torrence, C. and Compo, G.P., 1998. A Practical Guide to Wavelet Analysis. Bulletin of the American Meteorological Society, 79(1), pp. 61-78. https://doi.org/10.1175/15200477(1998)079<0061:APGTWA>2.0.CO;2

15. Zhegulin, G. V., 2016. Using Wavelet Analysis to Assess the Relationship of Hydrological and Hydrooptical Oscillations in the Range of Internal Waves according to In Situ Observations in the White Sea. Fundamentalnaya i Prikladnaya Gidrofizika, 9(3), pp. 48-56 (in Russian).

16. Grinsted, A., Moore, J.C., and Jevrejeva, S., 2004. Application of the Cross Wavelet Transform and Wavelet Coherence to Geophysical Time Series. Nonlinear Processes in Geophysics, 11(5/6), pp. 561-566. https://doi.org/10.5194/npg-11-561-2004

17. Kunsch, H.R., 1989. The Jackknife and the Bootstrap for General Stationary Observations. The Annals of Statistics, 17(3), pp. 1217-1241. doi:10.1214/aos/1176347265

18. Schulte, J.A., Najjar, R.G., and Lee, S., 2017. Salinity and Streamflow Variability in the MidAtlantic Region of the United States and Its Relationship with Large-Scale Atmospheric Circulation Patterns. Journal of Hydrology, 550, pp. 65-79. https://doi.org/10.1016/j.jhydrol.2017.03.064

19. Schulte, J., 2019. Higher-order Wavelet Analysis. Available at: http://www.mathworks.com/matlabcentral/fileexchange/54671-higher-order-wavelet-analysis [Accessed: 29 December 2019].

20. Zimin, A.V., 2018. Sub-tidal Processes and Phenomena in the White Sea. Moscow: GEOS, 220 p. (in Russian).

21. Zimin, A.V., Romanenkov, D.A., Kozlov, I.E., Chapron, B., Rodionov, A.A., Atadjanova, O.A., Myasoedov, A.G. and Collard, F., 2014. Short-Period Internal Waves in the White Sea: Operational Remote Sensing Experiment in Summer 2012. Issledovanie Zemli iz Kosmosa, 3, pp. 41-55. https://doi.org/10.7868/S0205961414030087 (in Russian).

22. Phillips, O.M., 1977. The Interaction of the Waves. In: S. Leibovich and A. R. Seabass, Eds., 1974. Nonlinear Waves. Ithaca, NY: Cornell University Press, 1974. pp. 186-205.

23. Saprykina, Ya.V., Shtremel, M.N. and Kuznetsov, S.Yu., 2017. On the Possibility of Biphase Parametrization for Wave Transformation in the Coastal Zone. Oceanology, 57(2), pp. 253264. https://doi.org/10.1134/S0001437016060126

24. Zhegulin, G.V., Zimin, A.V. and Rodionov, A.A., 2016. Analysis of the Dispersion Dependence and Vertical Structure of Internal Waves in the White Sea in Experimental Data. Fundamentalnaya i Prikladnaya Gidrofizika, 9(4), pp. 47-59 (in Russian).

25. Kozlov, I., Romanenkov, D., Zimin, A. and Chapron, B., 2014. SAR Observing Large-Scale Nonlinear Internal Waves in the White Sea. Remote Sensing of Environment, 147, pp. 99-107. https://doi.org/10.1016/j.rse.2014.02.017

26. Saprykina, Ya.V., Kuznetsov, S.Yu. and Divinskii, B.V., 2017. Influence of Processes of Nonlinear Transformations of Waves in the Coastal Zone on the Height of Breaking Waves. Oceanology, 57(3), pp. 383-393. https://doi.org/10.1134/S0001437017020187 
27. Elgar, S. and Guza, R.T., 1985. Observation of Bispectra of Shoaling Surface Gravity Waves. Journal of Fluid Mechanics, 161, pp. 425-448. doi:10.1017/S0022112085003007

28. Elgar, S. and Guza, R.T., 1986. Nonlinear Model Predictions of Bispectra of Shoaling Surface Gravity Waves. Journal of Fluid Mechanics, 167, pp. 1-18. doi:10.1017/S0022112086002690

About the authors:

Gleb V. Zhegulin, Junior Research Associate, Shirshov Institute of Oceanology of RAS, (36 Nakhimovsky Prospekt, Moscow, 117997, Russian Federation), Scopus Author ID: 57195070290, gleb-jegulin@rambler.ru

Aleksey V. Zimin, Chief Research Associate, Shirshov Institute of Oceanology of RAS, (36 Nakhimovsky Prospekt, Moscow, 117997, Russian Federation), professor of Saint-Petersburg State University (7/9 Universitetskaya Naberezhnaya, Saint-Petersburg, 199034, Russian Federation), WoS ResearcherID: C-5885-2014, Scopus Author ID: 55032301400, zimin2@mail.ru

Contribution of the co-authors:

Gleb V. Zhegulin - selection and analysis of literature, pitching of the paper idea, preparation of the paper text, creation of drawings

Aleksey V. Zimin - selection and analysis of literature, preparation and analysis of field data, critical analysis of the manuscript, addition and revision of the paper text

All the authors have read and approved the final manuscript.

The authors declare that they have no conflict of interest. 